\title{
Service-Oriented Layered Architecture for Smart Home
}

\author{
Muhammad Waqar Aziz \\ Department of Software Engineering, Faculty of Computing, Universiti Teknologi \\ Malaysia, Skudai, 81310, Malaysia \\ wamuhammad2@live.utm.my
}

\begin{abstract}
The successful implementations of the concepts of Service-Oriented Computing (SOC) in smart home in the recent years and the advantages it provide in the smart home development have proved its importance. However, efforts are still needed for service-oriented design of smart home, especially at the architectural level. Although a number of layered architectures are proposed for smart home in the past, most of them are related to middleware or hardware architecture design or explain the implementation details. A three-layered service-oriented architecture for smart home is proposed in this paper. On the one hand, the proposed architecture provides the facility to model the smart home in terms of devices and the services they provide, while on the other it provides the mean of communication to heterogeneous devices present in a smart home. To show the effectiveness of the proposed architecture, it was applied on "Food cooking and ordering" scenario in a smart home. The results showed that the proposed architecture helped in the increased understandability of the smart home design.
\end{abstract}

Keywords: Service-Oriented Computing, software architecture, layered architecture, smart home

\section{Introduction}

In a smart home the electrical devices are connected together, work automatically and serve its inhabitants [1-4]. For example, when a landline call is received while watching the $\mathrm{TV}$, the TV will automatically reduce the volume [1]. The fundamental problem of smart home is device, vendor, communication protocols and technology heterogeneity $[1,5]$. Other problems caused due to traditional server-centric architecture of a smart home are limited interoperability, scalability, extensibility [1], reliability and load concentration [6]. The use of Service-Oriented Computing (SOC) for smart home can solve these problems $[1,7,8]$.

The basic idea of SOC is that a service has defined interfaces through which the calling application can call and use them. The calling application does not know about the internal details of the service, whereas the service does not know about the calling application. This idea of SOC can be used in smart home, where the functionalities provided by devices can be represented as services. In smart home, each device shows its services and their interfaces, thus allowing other devices to use its services. This loosely-coupled connection between the devices allows them to work autonomously, while communicating in a standard way with each other [8].

Previously, SOC has been applied in smart home domain $[1,2,5,16]$, where devices appear as understandable services. In this way, the hardware and communication details of a device are abstracted from other devices. However, most of the existing work is concerned 
with the direct implementation of service-oriented concepts in smart home and very less attention has been given to design challenges especially the architecture design issues. Furthermore, the existing work in smart home architecture is mostly concerned with either middleware / hardware architecture design or provide the implementation details. It means that the existing architectures for smart home lack in providing any modeling mechanism and schematizing the system in terms of devices and their services.

Currently, service-oriented concepts are implemented directly for smart home and very little efforts have been made on systematic analysis and modeling. However, to fully utilize SOC and obtain more benefits, attention has to be given to service analysis and design for smart home. The first step in this regard can be to propose a service-oriented layered architecture as the service-oriented analysis process is concerned with identifying the services and building the service layers [9]. Moreover, the key SOA characteristics such as loose coupling can be achieved by placing the services into different layers of abstraction. To fulfill these needs, a three layered architecture for smart home is presented in this paper.

In contrast to existing smart home architectures, the proposed architecture does not provide the middleware / hardware or implementation details. Instead it provides a schematic way of representing the system in terms of the devices present in the smart home and the services they provide. In this regard, three layers were developed in order to separate the physical hardware devices on one layer from the application software on the other layer. The intermediate layer hides the physical hardware devices from the application layer by providing software services. This is to enable application software to view hardware devices as a set of services.

Furthermore, with the help of these layers the proposed architecture also provides an interaction mechanism between the heterogeneous devices. The services in the service layer allow the hardware devices to interact with each other (as explained in Section 3). Moreover, the services are classified in different categories to not only distinguish them but to make the overall system easier to understand. Besides separation of concern, categorizing the services of smart home in a layered architecture provides a number of other benefits:

1) Categorizes the design problems and identifies the design patterns to solve these problems [10]

2) Depicts the overall system as a set of services aligned with the devices providing these services (service providers)

3) Provides a logical service-based view of the physical devices

4) Separation of different types of services, such as, the application-specific services (software services) and services representing device functionality (real-world services)

5) Provides the interoperability between devices in terms of real-world services interacting with each other

6) Abstracts the physical devices from application software

7) Sets the basis for developing software architecture

8) Provides a way of integrating the physical devices with application software

9) Can be used in the service identification process [11]

10) Allows the direct communication between devices without any server (explain in Section 3)

The paper is organized as follows: Section 2 provides the methodology of proposing the layered architecture, which is followed by the details of the proposed service layered architecture in Section 3. Section 4 provides the details of implementing the proposed architecture in the smart home case study. The related work is provided in Section 5, while 
the discussion on the proposed architecture is provided in Section 6. Finally, the paper is concluded in Section 7.

\section{Methodology}

SOA provides an abstract view of a layered architecture of services aligned with the business processes. The same layered architectural view can be depicted by when it is used for smart home. Therefore using this idea, the layered approach was followed in the proposed architecture where the physical devices are abstracted from the application software. In this regard, the existing literature on layered architecture in SOC, embedded systems and cyber physical systems domain has been studied. A particular focus was given to systematic literature review on realizing SOA for cyber-physical systems [13].

\section{The proposed service-oriented proposed architecture}

The proposed architecture for smart home is divided into three layers: device layer, service layer and application layer as shown in Figure 1. The explanation of these layers is as follows:

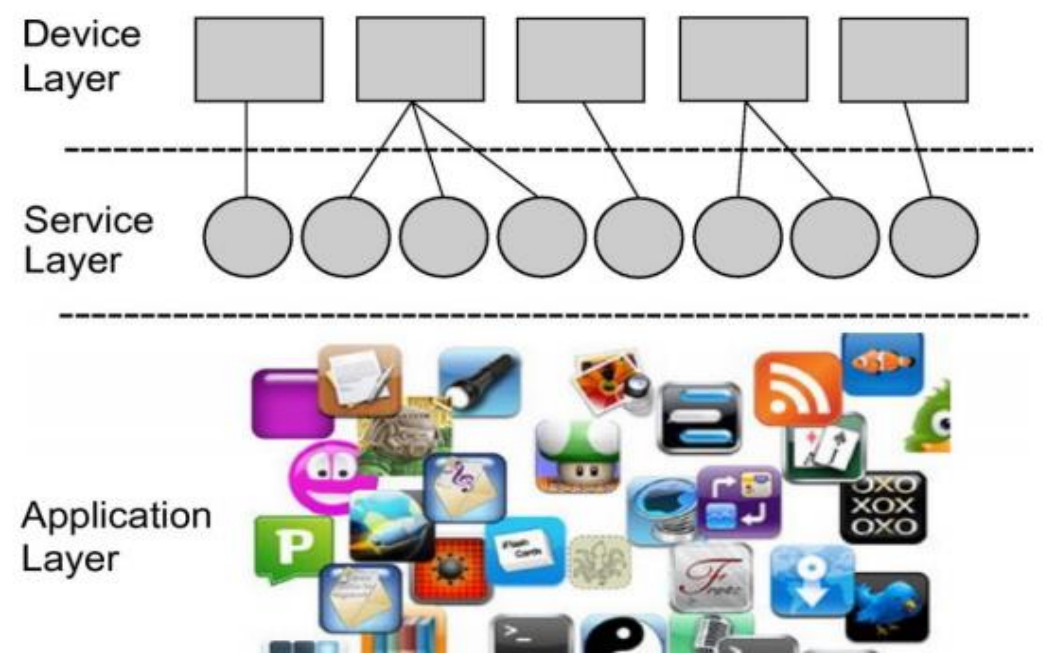

Figure 1. The Proposed Layered Architecture

\subsection{Device Layer}

This layer contains all physical hardware devices present in the system. It is assumed that each device in the smart home has some storage to store the operating system, a microcontroller, network interface, sensors and/or actuators. In addition, each device has one or more device control interfaces that can be accessed by software. These interfaces are wrapped as a method of service in the service layer, for example, a button with 'onoff' interfaces have corresponding methods in the service at the service layer.

The physical characteristics of the devices, such as device ID, location, processing power, memory and battery status etc., and the services they offer are useful in selecting the appropriate device. For simplicity these characteristics are not included and displayed in the proposed architecture; rather they are stored separately in a device registry. During the device selection process, the proposed three layered architecture can be used to portray the overall view of the system in terms of service request and its response. Thus, it makes the process of 
service/device selection easy to understand. This is shown with the help of an example in Figure 2, where a proper service/device discovery and selection mechanism is required. The service discovery and selection mechanism is supposed to provide the device information that is providing the requested service. However, the discovery, selection and orchestration of the services/devices, is a research topic on its own and is beyond the scope of this paper.

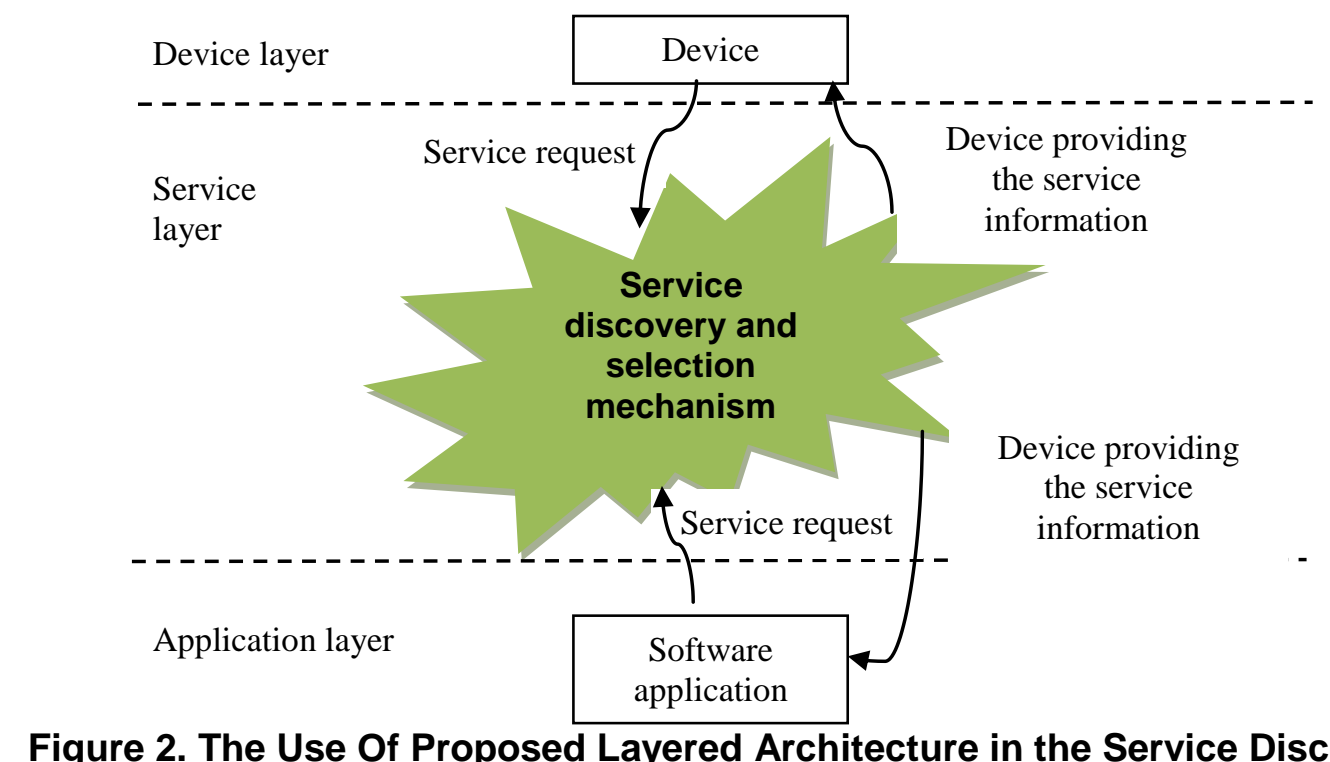

Figure 2. The Use Of Proposed Layered Architecture in the Service Discovery Process

The devices do not directly communicate with each other; rather the communication is via services present in the service layer: in order to use the services provided by other devices, a device has to use its own services. Thus, a common and standard way of communication, through services, is made possible between all heterogeneous devices while hiding the device-specific details.

\subsection{Service layer}

The services provided by the devices are depicted in this layer. This intermediate layer abstracts the physical hardware devices from the application layer. Thus, it enables the application software to view hardware devices as a set of services. In this work, the service is categorized into the following types: application service, functional service, control service, external service and device service, as shown in Figure 3. All these types are supposed to be atomic in nature that can be combined together into a composite service. For simplicity, an atomic service is represented as a circle/oval in the proposed architecture, with a special sign representing the different service types (see Figure 5). On the other hand, the composite service is represented as a big circle/oval having two or more atomic services. The detail of these different types of services is as follows.

The application service is a service that interacts with the application logic provided or derived from a technology platform such as message sending/receiving services provided by the service provider, order processing service provided by the application software of a retail store etc. The functional service represents the functional logic i.e., the functionality provided by a device such as on/off service, dim/full service and so on. In simple words, the functional service abstracts the device-specific raw data to and from 
sensors/actuators into meaningful information (in terms of service interfaces) and provides this information to other services and/or to the application layer. The control service defines and controls the process of service orchestration and is a mandatory part of a composite service. The external service represents a functionality provided by third party that is outside of the system, whereas the device service represents a physical device involved in the process that do not provide any service but uses the services of other devices.

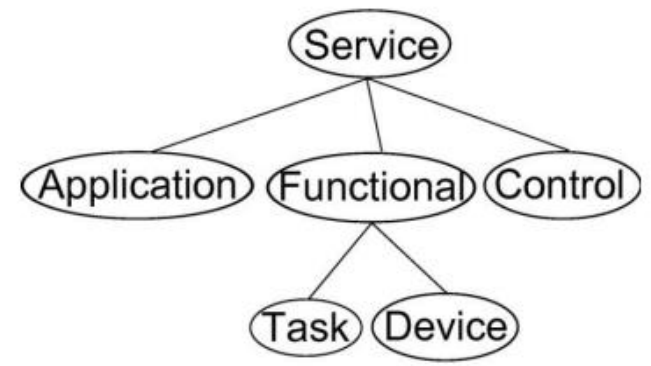

Figure 3. Proposed Service Categories

\subsection{Application layer}

The user can control the devices present in smart home through the application layer, i.e., it provides the interface to the user to control the devices. The application layer contains the software applications ranging from those present in different hardware devices to interact and control the smart home devices such as PDA, cell phone etc., to the enterprise applications that are used during device processes such as an order processing system of a retail store, to general web applications such as those providing email and SMS services.

These software applications can be provided by the in-house software using some local networking ability or by third parties over the Internet. Despite the development, use and selection of these software applications are outside the scope of this work, they are included in the proposed architecture to completely represent the service request and response flow within of the smart home.

\section{Food Cooking and Ordering Scenario}

In order to show the applicability of the proposed layered architecture, "Food cooking and ordering" scenario was considered in the smart home. Out of the smart home devices, shown in Figure 3, the considered scenario included television (TV), oven and a fridge. The "Food cooking and ordering" scenario in the smart home was defined as follows: Cooking message is sent from the oven to the user's cell phone and TV once the food is ready. Similarly, the fridge can send a message to the user and to the retail store to order the food item. The devices in the smart home and the application software were modeled and classified into three layers based on the proposed service-oriented layered architecture, as shown Figure 4. 


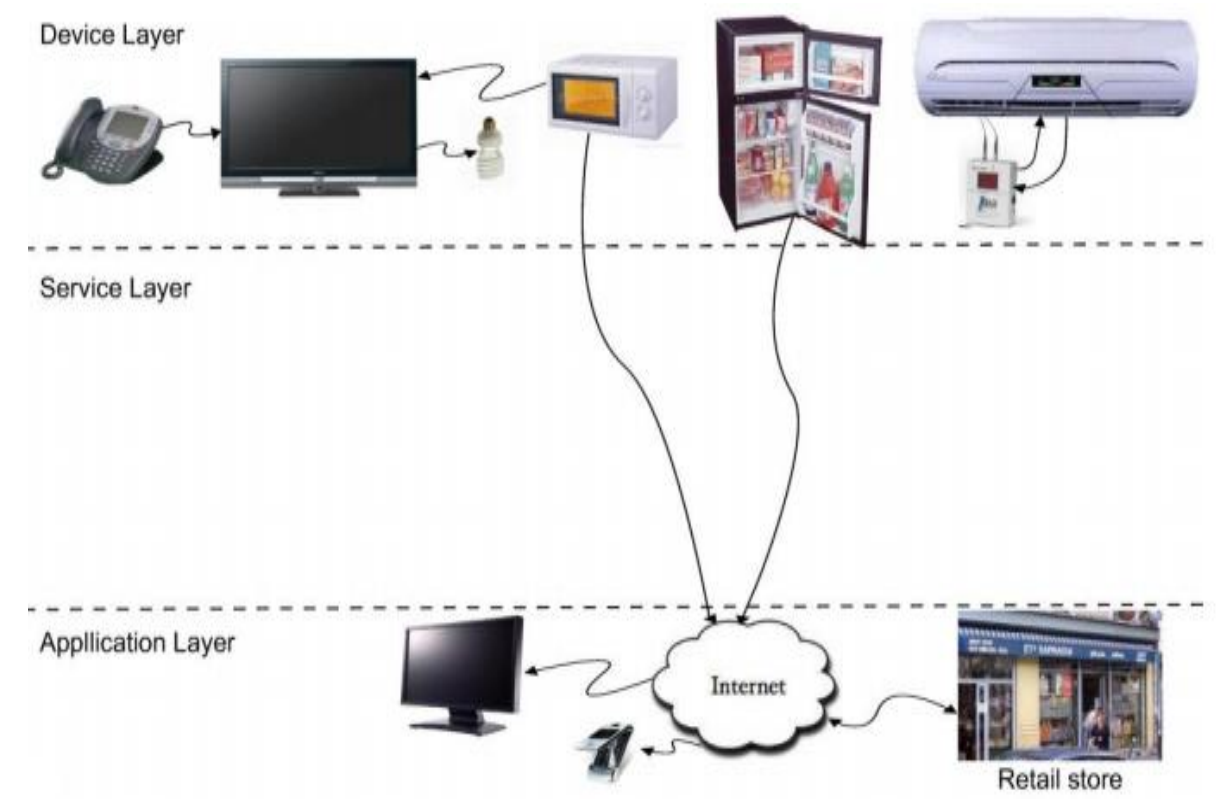

Figure 4. Smart Home Devices in the Proposed Layered Architecture

A bunch of services, identified with the help of our previously defined service identification guideline [11], was classified into the proposed service types and then modeled into the proposed architecture, as shown in Figure 5.

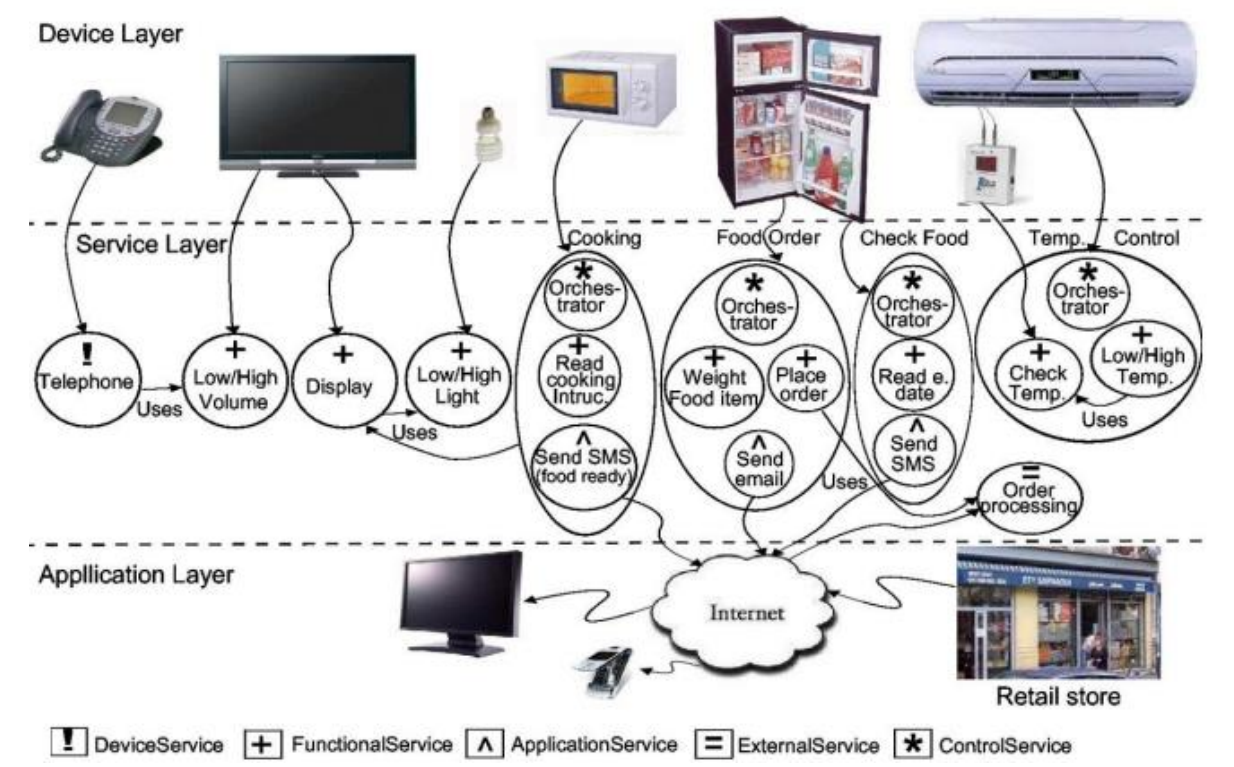

Figure 5. Services and Devices in the Proposed Layered Architecture

The identified services, as shown in Figure 5, were categorized into Device, Functional, Application, External and Control services. This categorization is presented in Table 1, along with the composite services. 
Table 1. Service Classification According to the Proposed Categories

\begin{tabular}{|l|l|}
\hline \multicolumn{1}{|c|}{ Category } & \multicolumn{1}{c|}{ Services } \\
\hline Device Service & Telephone \\
\hline Functional Service & $\begin{array}{l}\text { Low/high Volume, Display, Low/high light, } \\
\text { Read cooking Instruc., Weight Food item, Place Order, Tread } \\
\text { expiry date, Check Temp. Low/high Temp. }\end{array}$ \\
\hline Application Service & Send SMS, send e-mail \\
\hline External Service & Order Processing \\
\hline Composite Service & Cooking, Food order, Check Food, Temp. control \\
\hline
\end{tabular}

The distribution of services of the smart home case study along the layers in the proposed architecture is shown in Table 2.

Table 2. Service Distribution in Different Layers

\begin{tabular}{|l|l|}
\hline Layers & Services \\
\hline Control layer & Orchestrator \\
\hline Functional Layer & $\begin{array}{l}\text { Telephone, } \\
\text { Low/high Volume, Display, Low/high light, } \\
\text { Cooking, Food order, Check Food, Temp. control }\end{array}$ \\
\hline Application layer & Send SMS, send e-mail, Order Processing \\
\hline
\end{tabular}

\section{Related Work}

The existing work on smart home architecture can be roughly classified into three categories: architectures describing the middleware (middleware architecture); architectures describing the hardware (hardware architecture) and the architectures describing the implementation details (implementation architecture). Following are few examples of these classifications: the smart home architecture [17] is a middleware architecture that provides an overview of the middleware in three layers. However, it focuses on service composition in the smart home. On the other hand, the hardware architecture of the smart devices provided in [5, 16] describes the hardware and software components of the smart devices. Similarly, the service-oriented architecture for home networks [1] is focused on the implementation details, i.e., the OSGi network. These existing works on smart home architectures provide only the description of certain aspects (as mentioned in the above categories) of the design and do not provide the mean to model the devices and the services they provide.

Besides these classifications, there are some smart home architectures present in the literature focusing on a particular area. For example, a three layered cloud architecture is provided [15] to extend the cloud applications in the smart home domain. However, the architecture is particularly for cloud computing, is conceptual and requires implementation and improvements. Likewise, a three layered service architecture is proposed for cyber physical systems [12], however in the architecture [12] network layer is used between device and service layers while there is no application layer.

The service is classified into different types in [14] but from the location- and contextawareness point of view and hence this classification is not valid for service-oriented modeling of the smart home.

Besides SOC, there are other solutions present in the literature that focus on software architecture for modeling of the smart home. For example, Wei and Yun-Wei [3] use AADL to model the smart home, but the modeling is focused more on the physical level than the 
software. However, it provides a good idea of whole dividing the architecture into three separate layers.

\section{Discussion}

The proposed service layer architecture provides the facility to model the smart home, ease of understanding, way of communication and the aid in service discovery and selection process. Moreover, the service is categorized in different types in this work. All these points are discussed in the following.

Modeling facility: The proposed architecture provides the means of modeling the smart home i.e., an overall picture of the hardware devices, the provided functionality and the application software present in the smart home can be modeled (see e.g., Figure 5 ). The model thus developed, based on the proposed architecture, can be helpful in the design of smart home where it can be used as a design artifact.

Ease of understanding: The visual representation of the smart home, provided by the proposed architecture, leads to an easy understanding of the functional behavior of the smart home in terms of offered services and their interactions. As a result, this visual representation can be easily used and interpreted by the group of people working on smart home (e.g., analyst, designers, programmers, stakeholders etc.) during its analysis and design. Thus, the overall analysis and design process of the smart home is simplified.

Way of communication: In the proposed architecture, the vendor specific devices do not directly communicate with each other. Instead the communication between devices is via the services they provide. Thus, a common and standard way of communication between heterogeneous devices is made possible and the device-specific details are hidden from the application software.

Aid in service discovery and selection process: The proposed layered architecture is useful in service discovery and selection process, as it provide the visual aid (see Figure 2 ) to understand the overall flow of service request and response in the smart home.

Service categorization: The service categorization proposed in this work makes it possible to distinguish between different types of services. On the one hand, this categorization clarifies the design and allows the designers to separate services of different types and to focus on the required type; on the other they define a particular role of a service (control, functional and so on, as shown in Figure 5). These roles provide validation checks of the design e.g. the Device service cannot be Functional or Control service at the same time and vice versa. Alternatively, an Application service can be an External service.

\section{Conclusion}

In this paper service-oriented architecture for smart home is proposed and its three layers are illustrated. The proposed architecture provides a way to model the smart home in terms of the devices and their services. In addition, different categories of services are specified. The proposed layered architecture and the specified service categories allow modeling the smart home on the one hand and distinguishing between different types of services. The proposed architecture is applied on the "Food cooking and ordering scenario" in a smart home. The proposed architecture can be useful in service identification during service-oriented design of the smart home. Furthermore, the proposed architecture is supposed to be a part of the larger system analysis and design process for smart home. The future directions of this 
research is to propose a complete systematic analysis and design process of smart home that include the proposed layered architecture also.

\section{Acknowledgements}

We would like to thank the Malaysia Ministry of Higher Education (MOHE) for sponsoring the research and Universiti Teknologi Malaysia (UTM) for providing the facilities and support for the research.

\section{References}

[1] L. Ngo, "Service-oriented architecture for home networks", TKK T-110.5190 Seminar on Internetworking 2007-3-4/5, (2007).

[2] V. Ricquebourg, D. Menga and D. Durand, “The smart home concept: our immediate future”, First IEEE Int. Conf. on E-Learning in Industrial Electronics, (2006).

[3] S. Wei and D. Yun-Wei, "On designing and verifying smart home embedded software model based on AADL", Second Int. Conf. on Future Generation Communication and Networking, 2008, FGCN '08, (2008).

[4] W. Jiankun, H. Linpeng and W. Dejun, "R-OSGi-based architecture of distributed smart home system", IEEE Trans. Consum. Electron, vol. 54, no. 3, (2008), pp. 1166-1172.

[5] T. Kawashima, M. Jianhua and H. Runhe, "GUPSS: A gateway-based ubiquitous platform for smart space", Int. Conf. on Computational Science and Engineering, 2009, CSE’09, (2009).

[6] M. ONakamura, H. Igaki and H. Tamada, "Implementing integrated services of networked home appliances using service oriented architecture", Second Int. Conf. on Service Oriented Computing, ACM, New York, NY, USA, (2004).

[7] W. Chao-Lin, L. Chun-Feng and F. Li-Chen, "Service-oriented smart-home architecture based on OSGi and mobile-agent technology”, IEEE Trans. Syst. Man Cybern. C, Appl. Rev., vol. 37, no. 2, (2007), pp. 193-205.

[8] J. Bourcier, A. Chazalet and M. Desertot, "A dynamic-SOA home control gateway", IEEE Int. Conf. on Services Computing, SCC'06, (2006).

[9] T. Erl, "Service-Oriented Architecture: Concepts, Technology, and Design", NJ, USA: Prentice Hall PTR, (2005).

[10] C. Mauro, J. M. Leimeister and H. Krcmar, "Service Oriented Device Integration - An Analysis of SOA Design Patterns", 43rd Hawaii International Conference on System Sciences (HICSS-43), Poipu, Kauai, Hawaii, (2010) January 5-8, pp. 1-10.

[11] R. Mohamad, M. W. Aziz, D. N. A. Jawawi, M. Ghazali, M. Z. Arbaie and N. Ibrahim, "Service identification guideline for developing distributed embedded real-time systems", IET Software, vol. 6, no. 1, (2012), pp. 74-82

[12] W. Xiao-Le, H. Hong-Bin, D. Su and C. Li-Na, "A Service-Oriented Architecture Framework for CyberPhysical Systems", Qian Z., Cao L., Su W., Wang T. and Yang H. (Eds.). Recent Advances in Computer Science and Information Engineering, Berlin, Heidelberg: Springer, vol. 126, (2012), pp. 671-676.

[13] D. D. Hoang, H.-Y. Paik, C.-K. Kim, "Service-Oriented Middleware Architectures for Cyber-Physical Systems", IJCSNS International Journal of Computer Science and Network Security, vol. 12, no, 1, (2012), pp. 79-87.

[14] Y. Uhm, Z. Hwang, M. Lee, Y. Kim and S. Park, "Service Reconstruction for Improving Performance using Classified Service Pattern and a Session Manager-based Architecture in Smart Homes", 5th IEEE Consumer Communications and Networking Conference (CCNC) Las Vegas, (2008) January 10-12, pp. 326-330.

[15] Y. Yang, Z. Wei, D. Jia, Y. Cong and R. Shan, "A Cloud Architecture Based on Smart Home", Second International Workshop on Education Technology and Computer Science, (2010).

[16] M. Nakamura, H. Igaki, H. Tamada and K.-I. Matsumoto, '"Implementing Integrated Services of Networked Home Appliances Using Service Oriented Architecture”, 2nd International Conference on Service Oriented Computing (ICSOC'04), New York, (2004) November 15-19.

[17] E. Kaldeli, E. U. Warriach, J. Bresser, A. Lazovik and M. Aiello, "Interoperation, composition and simulation of services at home", 8th Int. Conf. on Service Oriented Computing (ICSOC-10), Springer, vol. LNCS 6470, (2010), pp. 167-181. 


\section{Authors}

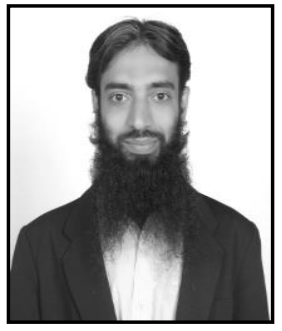

Muhammad Waqar Aziz, has received Doctor of Philosophy (Computer Science) from Universiti Teknologi Malaysia, MS-Software Engineering from City University of Science and Technology and MScComputer Science from University of Peshawar. Previously, he has been a Lecturer at Institute of Management Studies, University of Peshawar for almost eight years. The research areas of his interest are software modeling, service-oriented computing, and embedded real-time software development. 